\title{
Inversion, error analysis, and validation of GPS/MET occultation data
}

\author{
A. K. Steiner ${ }^{1}$, G. Kirchengast ${ }^{1}$, H. P. Ladreiter $^{2}$ \\ ${ }^{1}$ Institut für Meteorologie und Geophysik, Karl-Franzens-Universität Graz, Graz, Austria \\ ${ }^{2}$ Institut für Weltraumforschung, Österr. Akademie der Wissenschaften, Graz, Austria
}

Received: 16 February 1998 / Revised: 27 May 1998 / Accepted: 9 June 1998

\begin{abstract}
The global positioning system meteorology (GPS/MET) experiment was the first practical demonstration of global navigation satellite system (GNSS)-based active limb sounding employing the radio occultation technique. This method measures, as principal observable and with millimetric accuracy, the excess phase path (relative to propagation in vacuum) of GNSS-transmitted radio waves caused by refraction during passage through the Earth's neutral atmosphere and ionosphere in limb geometry. It shows great potential utility for weather and climate system studies in providing an unique combination of global coverage, high vertical resolution and accuracy, long-term stability, and all-weather capability. We first describe our GPS/MET data processing scheme from excess phases via bending angles to the neutral atmospheric parameters refractivity, density, pressure and temperature. Special emphasis is given to ionospheric correction methodology and the inversion of bending angles to refractivities, where we introduce a matrix inversion technique (instead of the usual integral inversion). The matrix technique is shown to lead to identical results as integral inversion but is more directly extendable to inversion by optimal estimation. The quality of GPS/ MET-derived profiles is analyzed with an error estimation analysis employing a Monte Carlo technique. We consider statistical errors together with systematic errors due to upper-boundary initialization of the retrieval by a priori bending angles. Perfect initialization and properly smoothed statistical errors allow for better than $1 \mathrm{~K}$ temperature retrieval accuracy up to the stratopause. No initialization and statistical errors yield better than $1 \mathrm{~K}$ accuracy up to $30 \mathrm{~km}$ but less than $3 \mathrm{~K}$ accuracy above $40 \mathrm{~km}$. Given imperfect initialization, biases $>2 \mathrm{~K}$ propagate down to below $30 \mathrm{~km}$ height in unfavorable realistic cases. Furthermore, results of a statistical validation of GPS/MET profiles through comparison with atmospheric analyses of the European
\end{abstract}

Correspondence to: A. K. Steiner
Centre for Medium-range Weather Forecasts (ECMWF) are presented. The comparisons indicate the high utility of the occultation data in that very good agreement of upper troposphere/lower stratosphere temperature (better than $1.5 \mathrm{~K} \mathrm{rms,}<0.5 \mathrm{~K}$ bias) is found for a region (Europe + USA) where the ECMWF analyses are known to be good, but poorer agreement for a region (Southern Pacific) where the analyses are known to be degraded.

Key words. Atmospheric composition and structure (pressure, density and temperature) - Meteorology and atmospheric dynamics (instruments and techniques) Radio science (remote sensing)

\section{Introduction}

The application of the radio occultation method for the study of planetary atmospheres enables the determination of height profiles of refractivity, and the derivation of further parameters such as density, pressure, temperature and tropospheric water vapor pressure in the neutral atmosphere and electron density in the ionosphere. The principle of this remote sensing technique is based on the influence of an atmospheric or ionospheric refractivity field on electromagnetic waves propagating through the field in limb sounding geometry.

Initially the radio occultation technique was applied to the investigation of the atmospheres of Mars and Venus (Fjeldbo and Eshleman, 1965; Phinney and Anderson, 1968; Fjeldbo et al., 1971), and in the following years also to those of the outer planets and their satellites (e.g., Lindal et al., 1983, 1985). There, a transmitter on a spacecraft far outside the atmosphere of the respective planet broadcasts radar waves which are received by a station on the Earth. If the trajectory is such that the spacecraft moves behind the planet, as 
viewed from the Earth, the electromagnetic ray will pass tangentially through the atmosphere, and will be occulted at the planetary limb. The radio signal undergoes slowing and bending during its propagation through the atmosphere and ionosphere, and the receiver collects the respective signal samples as a function of height. Vertical profiles of atmospheric and ionospheric parameters are determined from the observed phase changes and amplitude variations.

Since the first proposal for remote sensing of the terrestrial atmosphere using a radio occultation technique in the 1960s (Fishbach, 1965; Lusignan et al., 1969) many theoretical and experimental investigations have been performed (e.g., Gorbunov, 1988). The successful installation of the US Global Positioning System (GPS) satellites during the last decade, and of the Russian GLONASS system more recently, and the accurate determination of satellite positions and velocities feasible in the meantime allow now the practical application of the radio occultation method to sense the terrestrial atmosphere. Early proposals for this modern application of the method have been outlined by Gurvich and Krasil'nikova (Russian original 1987; English translation 1990) and Yunck et al. (1988).

The method was first tested with the GPS Meteorology (GPS/MET) "proof-of-concept" experiment, which was led by the University Corporation for Atmospheric Research (UCAR) (Ware et al., 1996). According to the characteristic geometry of such GPS/GLONASS-based occultation measurements, the GPS/MET experiment provided occultation data gathered in an active limb sounding mode based on a satellite-to-satellite radio link. After its launch in April 1995 aboard the small research satellite MicroLab-1 into a near-circular Low Earth Orbit (LEO), the high-performance dual-frequency GPS/MET receiver tracked typically more than a hundred occultation events per day, i.e., collected excess phase and amplitude data during the setting of the GPSto-LEO radio link through the Earth's atmosphere towards the Earth's limb. The mission, collecting data until mid 1997, was the first successful phase of a plan to develop a worldwide constellation of LEO satellites, which will operationally provide a globally distributed data set of fundamental atmospheric and ionospheric variables.

This study employs the GPS/MET occultation data to advance inversion techniques as well as the knowledge on error characteristics, and it contributes to the validation of GPS/MET data. It is organized as follows. A brief description of the GPS/MET data is given in Sect. 2. Our GPS/MET data analysis procedure, with emphasis on the ionospheric correction methodology and the matrix inversion technique used within the retrieval chain, is described in Sect. 3. The accuracy of the retrieved atmospheric profiles (bending angle, refractivity, pressure, temperature) is analyzed and quantified in Sect. 4 with an error estimation analysis based on Monte-Carlo simulations. Results of a validation effort, where retrieved refractivity and temperature profiles are compared with atmospheric analyses produced by the European Centre for Medi- um-range Weather Forecasts (ECMWF), are presented in Sect. 5 .

\section{The GPS/MET data}

The LEO satellite MicroLab-1 orbited the Earth at an altitude of about $750 \mathrm{~km}$ with a period of about 100 min. The GPS network consists of (nominally) 24 satellites orbiting the Earth twice per day at an altitude of about $20000 \mathrm{~km}$. Each GPS satellite continuously transmits signals at two $L$-band carrier frequencies, $1575.42 \mathrm{MHz}(L 1)$ and $1227.6 \mathrm{MHz}(L 2)$, corresponding to wavelengths of $19.0 \mathrm{~cm}$ (L1) and $24.4 \mathrm{~cm} \mathrm{(L2),}$ respectively. An occultation event occurred each time, when the GPS satellite descended behind the Earth's atmosphere towards the Earth's surface limb as viewed from the aft-looking receiving antenna of the LEO satellite. Per day up to approximately 150 setting occultation events were processed, which were nearly arbitrarily distributed over the globe.

Each such occultation event has a typical duration of 1-2 min (spanning a height range up to $\sim 100 \mathrm{~km}$ ). Within this time the GPS/MET receiver collected measurements of excess phase paths (at $L 1$ and $L 2$ frequencies) at a sampling rate of $50 \mathrm{~Hz}$. When the Anti-Spoofing (A/S) encryption of the GPS signals was turned off, both $L 1$ and $L 2$ data were sampled at $50 \mathrm{~Hz}$ with equal performance. These have been termed the "Prime Time" data. In the normal operational mode of GPS, A/S is turned on, which causes a significantly noisier L2 signal, and requires code-less tracking techniques. Under these conditions, integration of $L 2$ phase data to rates lower than at $L 1$ (e.g., to $10 \mathrm{~Hz}$ or $1 \mathrm{~Hz}$ ) can reduce their noise. The topic of processing using reduced L2 sampling rates is addressed in Sect. 3.1.1.

A typical measurement profile for the neutral atmosphere starts at a height of about $100 \mathrm{~km}$ and shows phase delays of only about $1 \mathrm{~mm}$ at the height of the mesopause $(\sim 80 \mathrm{~km})$. However, as the signal tracking is successively performed through lower and thus denser layers of the atmosphere the delay increases, via about $20 \mathrm{~cm}$ at stratopause level and about $10 \mathrm{~m}$ at tropopause level, to about $1 \mathrm{~km}$ at the surface (about $700 \mathrm{~m}$ in a dry atmosphere and up to about $2 \mathrm{~km}$ in a humid atmosphere; see e.g., Melbourne et al., 1994).

For the following data analyses, we used GPS/MET "level 2 data" from October 1995, mainly October 20 and 21, which have been recorded during GPS/MET "Prime Time 3" (A/S-off conditions). The GPS/MET level 2 data files contain, in particular, $50 \mathrm{~Hz}$-sampled time series of the LEO and GPS satellite positions and velocities and of the excess phase paths $\left(\phi_{1}, \phi_{2}\right)$ at the two carrier frequencies $(L 1, L 2)$. The excess phase paths contain only ionospheric and atmospheric phase path contributions because the vacuum path length contributions have already been removed. Very good scientific overviews on the experiment together with a presentation of first scientific results of GPS/MET data analysis have been given by Ware et al. (1996) and Kursinski 
et al. (1996). Furthermore, detailed information on the experiment and the data can be found via the GPS/MET homepage (http://pocc.gpsmet.ucar.edu).

\section{GPS/MET data analysis}

The GPS/MET data processing chain from the measured phase data to the derivation of neutral atmospheric parameters can be divided into three steps (for each occultation event):

1. Calculation of the atmospheric bending angle profile from the observed $L 1 / L 2$ excess phase path time series (including dual-frequency ionospheric correction)

2. Retrieval of the refractive index profile from the atmospheric bending angle profile (the actual inversion)

3. Calculation of refractivity, density, pressure and temperature profiles based on the refractive index profile (assuming dry air)

\subsection{Bending angle calculation}

In the geometric optics approximation, a ray passing through the atmosphere behaves according to Fermat's principle of least time (e.g., Born and Wolf, 1980). The bending of the ray is described by Snell's law linking the bending to the vertical gradient of the refractive index of the atmosphere. The cumulative effect of the atmosphere along the path of propagation can be characterized by the total bending angle $(\alpha)$ as a function of the impact parameter $(a)$. The impact parameter is defined, assuming spherical symmetry, as the perpendicular distance between the center of the Earth (more precisely speaking, the center of local curvature at the perigee of the occultation ray) and the ray asymptote at the GPS or LEO satellite. Figure 1 illustrates the occultation geometry described.

The GPS transmitter corresponds to an electromagnetic point-source, the atmosphere acts like a weak inhomogeneous lens, and the LEO receiver consequently receives a Fresnel diffraction-limited signal. The

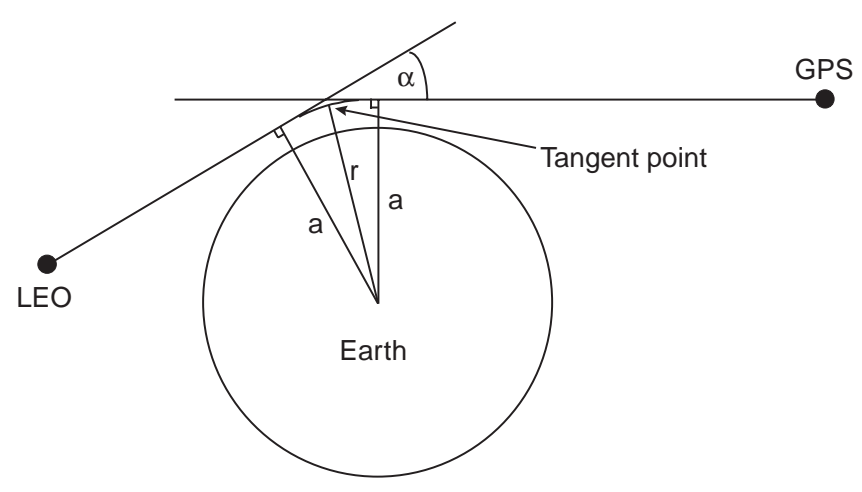

Fig. 1. GPS-LEO occultation geometry defining the atmospheric bending angle $\alpha$, the impact parameter $a$, and the radius to the ray tangent point (ray perigee), $r$ natural vertical resolution thus achieved is better than $1.5 \mathrm{~km}$ in the stratosphere and better than $0.5 \mathrm{~km}$ in the lower troposphere. The horizontal resolution is about $300 \mathrm{~km}$ along the GPS-to-LEO raypath and about $1.5 \mathrm{~km}$ perpendicular to the path (Melbourne et al., 1994; Kursinski et al., 1997). We do not consider diffraction effects in this study, i.e., measures for deconvolving diffractive effects in troposphere/tropopause excess phase data have not been taken, though they can be very useful and further enhance vertical resolution (see on this topic, e.g., Gorbunov et al., 1996).

In our data processing, first of all the level 2 excess phase time series are filtered (with runaway-data removal by 3-sigma level rejection and with sliding window averages over 5 data points) to reduce the noise and to smooth the data. Next, the $L 1 / L 2$ excess Doppler shifts are calculated by third order polynomial-fit differentiation of the $L 1 / L 2$ phase paths (with sliding fit over 7 data points); they are then available at approximately the mentioned minimal natural vertical resolution of about $0.5 \mathrm{~km}$. The respective bending angles $\alpha_{1}$ and $\alpha_{2}$ as functions of the impact parameters $a_{1}$ and $a_{2}$ are then calculated from the excess Doppler shifts and the given measurements of transmitter and receiver position and velocity vectors following Vorob'ev and Krasil'nikova (1994).

As the core of the removal of the ionospheric contribution to the atmospheric bending angle, the linear correction method of bending angles is applied (Vorob'ev and Krasil'nikova, 1994; Hocke, 1997) as follows

$\alpha(a)=\frac{f_{1}^{2} \alpha_{1}(a)-f_{2}^{2} \alpha_{2}(a)}{f_{1}^{2}-f_{2}^{2}}$,

where $\alpha_{1}$ and $\alpha_{2}$ need to be matched to the same impact parameter. This correction method provides significantly better results than the traditional linear correction of phases, as was stated by several theoretical studies (e.g., Vorob'ev and Krasil'nikova, 1994; Ladreiter and Kirchengast, 1996; Hocke, 1997). Recently, this was also confirmed practically by Hocke et al. (1997) by employing rigorous forward-inverse simulations based on atmospheric and ionospheric models and by analyzing GPS/MET data.

The three following subsections briefly address three specific relevant problems involved in bending angle determination, which include ionospheric correction of GPS data under A/S-on conditions (Subsect. 3.1.1), ionospheric correction in the troposphere (Subsect. 3.1.2), and upper-boundary initialization by add-on bending angle data being a necessary complement to the measured bending angles (Subsect. 3.1.3). The report by Hocke et al. (1997) furnishes a more detail discussion of these topics.

3.1.1 Evaluation of the effect of using reduced L2 sampling rates. The Anti-Spoofing (A/S) of the GPS L2 signal (i.e., the intentional encryption of the code modulation 
on $L 2$ ) forces us to employ code-less tracking techniques for dual-frequency GPS signal tracking, the penalty of which is significantly degraded signal-to-noise ratio and consequently enhanced phase noise. (The GLONASS system does not suffer from an A/S limitation, which is one attractive advantage of this complementary navigation satellite system.) It appears sensible under $\mathrm{A} / \mathrm{S}$-on conditions to perform the ionospheric correction with reduced initial $L 2$ sampling rate (of order $1 \mathrm{~Hz}$, to largely damp noise) compared to the $L 1$ rate (baseline sampling $50 \mathrm{~Hz}$ ).

We evaluated three methods for performing ionospheric correction given reduced $L 2$ sampling rate, in terms of the temperature accuracy they are capable of furnishing. For convenience, the reasonable assumption that the $L 2$ rate is an integer divisor of the $L 1$ rate has been made. The first straightforward method is to increase by interpolation the lower initial $L 2$ rate to the $L 1$ rate and then to apply the dual-frequency (bending angle) correction. The disadvantage of this method is that "smooth" data at $L 2$ are directly combined with $L 1$ data containing "high-frequency" variability sampled only at the higher rate.

In the second method, the $L 1$ rate is first smoothed to match the lower initial $L 2$ rate, and then a dualfrequency phase correction is performed at the lower $L 2$ rate. The resulting neutral atmospheric data are finally interpolated back to the higher $L 1$ rate, and the "highfrequency" variability sampled only at the higher rate is added. Unfavorably, the bending angle correction cannot be applied in this case.

The third method is formally very similar to the second one but allows bending angle correction. First one increases the lower initial $L 2$ rate to the higher $L 1$ rate by interpolation, then adds to these "smooth" highrate $L 2$ data the "high-frequency" phase variability sampled only at $L 1$, and finally applies the bending angle correction. This method turns out to be clearly superior and was implemented in our data processing chain (not further employed for the GPS/MET "Prime Time" data analysis, however).

The second and third method intrinsically assume that the "high-frequency" phase variability, sampled at $L 1$ only, is entirely due to the neutral atmosphere (with negligible ionospheric contribution). This assumption, which is quite reasonable for sampling rates $\geq 1 \mathrm{~Hz}$, allows to "transfer" the (non-dispersive) phase variability on the subsecond time scale from $L 1$ to $L 2$ as outlined.

Analyzing a few arbitrarily drawn GPS/MET occultation events, all three methods have been investigated for representative initial $L 1 / L 2$ sampling rate pairs of $50 \mathrm{~Hz} / 50 \mathrm{~Hz}$ (reference case), $50 \mathrm{~Hz} / 25 \mathrm{~Hz}, 50 \mathrm{~Hz} /$ $10 \mathrm{~Hz}$ and $50 \mathrm{~Hz} / 1 \mathrm{~Hz}$. The performance was evaluated in terms of differences of the retrieved temperature profiles $(\Delta T)$ with respect to the $50 \mathrm{~Hz} / 50 \mathrm{~Hz}$ reference case. Figure 2 illustrates, for one event, the results after application of the third method, the method of choice. This method has the potential to retain sub-Kelvin accuracy up to $30 \mathrm{~km}$ height even for a $50 \mathrm{~Hz} / 1 \mathrm{~Hz}$ sampling ratio.
3.1.2 Evaluation of ionospheric correction in the troposphere. The tropospheric refractivity field often exhibits significant small-scale variability mainly due to the presence of a highly variable moisture distribution and temperature inversions. Here, splitting of $L 1$ and $L 2$ raypaths incurred by passing the (dispersive) ionosphere on the way from the transmitter, causes these rays to often probe quite different local refractivity behavior at the different perigee heights reached (difference up to several $100 \mathrm{~m}$ ). Multipath propagation of the $L 1$ and $L 2$ rays caused by high refractivity gradients can further complicate the received signal. Under these conditions, straightforward linear combination of $L 1$ and $L 2$ data produces large, spurious errors in the resulting ionosphere-corrected tropospheric bending angle data. It is thus reasonable to consider alternatives to direct dualfrequency correction in the troposphere. Fortunately, ionospheric correction errors are much less relevant in the troposphere than higher up since the neutral atmospheric excess phase (and Doppler) largely exceeds the ionospheric one there (e.g., Hocke, 1997).

We evaluated three alternative methods, which all avoid direct use of the $L 2$ data below the tropopause level (defined at $12.5 \mathrm{~km}$ height for the evaluation, corresponding approximately to the $200 \mathrm{mbar}$ level). Above this level the bending angle correction was performed as usual. We note that no need for tropospheric $L 2$ data were an advantage also from the instrument point of view, since especially the tracking of the $L 2$ signal throughout the troposphere is very difficult to perform (see Hocke et al., 1997).

The first straightforward method is to switch, at the tropopause level, from the usual dual-frequency corrected bending angles to bending angles which are derived solely from the $L 1$ Doppler data (ignoring $L 2$ information). This intrinsically assumes that the ionospheric Doppler is negligible compared to the neutral one in the troposphere, a rather crude approximation.

The second method is to use $L 1$ Doppler data below the tropopause level having added a constant ionospheric Doppler estimate for each ray sample. This estimate is obtained immediately above the tropopause level and calculated using the $L 1-L 2$ differential Doppler. This intrinsically assumes the ionospheric Doppler contribution to be nearly constant over the whole troposphere, an improved approximation compared to the first method.

The third method, which gives the best results, is currently implemented in our data processing chain. Below the tropopause level the $L 2$ Doppler data are replaced by $L 1$ Doppler data, having added to each ray sample the ionospheric Doppler estimate extrapolated linearly downwards from the lower stratosphere. We obtain this estimate by using the averages of about 100 samples around $25 \mathrm{~km}$ and around $15 \mathrm{~km}$ as the basic two point estimates defining the linear ionospheric Doppler height dependence. This exploits the ionospheric Doppler information to an additional order, and assumes that it varies linearly over about $30 \mathrm{~km}$ width above the Earth's surface. The bending angle correction is then applied invoking these extrapolated $L 2$ Doppler data. 

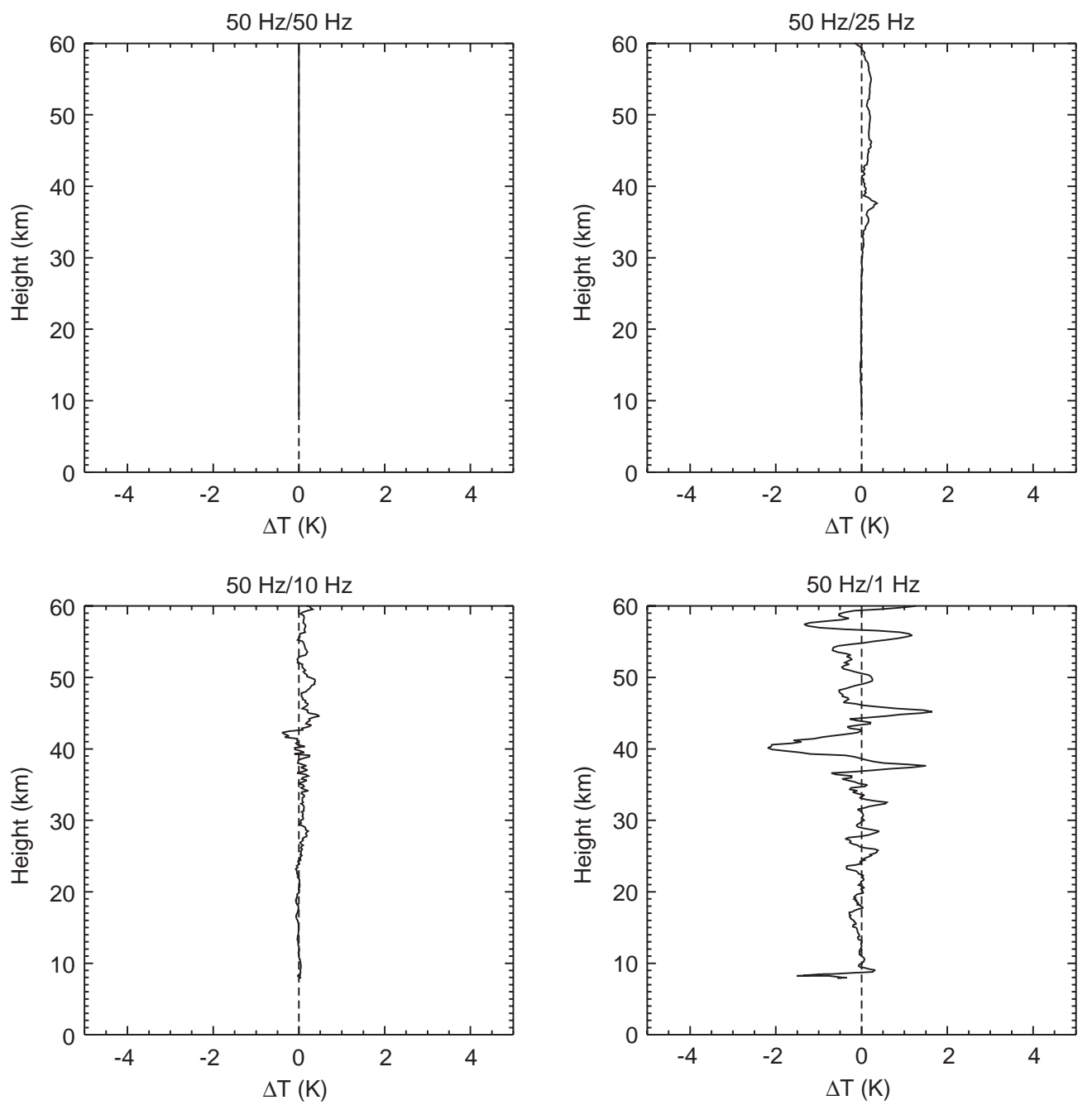

Fig. 2. The quality of ionospheric correction in case of reduced $L 2$ sampling rate is shown in terms of retrieved temperature differences relative to an $L 1 / L 2$ baseline case of $50 \mathrm{~Hz} / 50 \mathrm{~Hz}$. Our "method of choice" (see text) for different $L 1 / L 2$ sampling ratios of $50 \mathrm{~Hz} / 50 \mathrm{~Hz}$,

$50 \mathrm{~Hz} / 25 \mathrm{~Hz}, \quad 50 \mathrm{~Hz} / 10 \mathrm{~Hz}$ and $50 \mathrm{~Hz} / 1 \mathrm{~Hz}$ is illustrated. The primary data are from GPS/MET occultation event 80 on October $25,1995,09: 43 \mathrm{UT}$, located at $12.7^{\circ} \mathrm{N}$ and $179.3^{\circ} \mathrm{W}$

This third method, our method of choice, is illustrated for one GPS/MET event in Fig. 3. The upper two panels indicate, for the third and first method, that the choice of different methods can lead to differences in retrievals of $>1 \mathrm{~K}$. The bottom panel shows the raw ionospheric Doppler (ignoring a constant factor) throughout the tropo- and stratosphere together with the linear Doppler estimate used for the third method. It indicates that it was not meaningful to correct by direct $L 1 / L 2$ combination for the complex ionospheric Doppler fluctuations seen in the troposphere. Based on the case studies performed, the extrapolation method, requiring no $L 2$ data below the tropopause level at all, appears to be a sensible alternative for correcting tropospheric data. However, a more rigorous future study on this topic is certainly needed, since reliable ionospheric correction of tropospheric occultation data will be of high relevance during the period of high solar activity coming in the next years.
3.1.3 Statistical optimization approach. The signal-tonoise ratio in the measurement-derived bending angle becomes less than unity at heights above 40 to $50 \mathrm{~km}$, which necessitates upper-boundary initialization by addon bending angle data. In our data processing we therefore combine the GPS/MET bending angle data with a priori model bending angle data for the derivation of individual atmospheric profiles. Before this combination, data runaways are eliminated from the measured data (by 3-sigma level rejection), and a $\operatorname{Cos}^{2}$-Filter with height-dependent filter width is applied down to $30 \mathrm{~km}$ height ( 25 points-width above $40 \mathrm{~km}$, linearly decreasing to zero-width at $30 \mathrm{~km}$ ). Figure 4 compares a typical measured bending angle profile together with the bending angle profile obtained after combination with model data. In the example shown, the model bending angle was derived from the MSISE90 atmospheric model (Hedin, 1991). The combination controls the errors in the measured data at high altitudes and provides sensible initialization at the upper boun- 

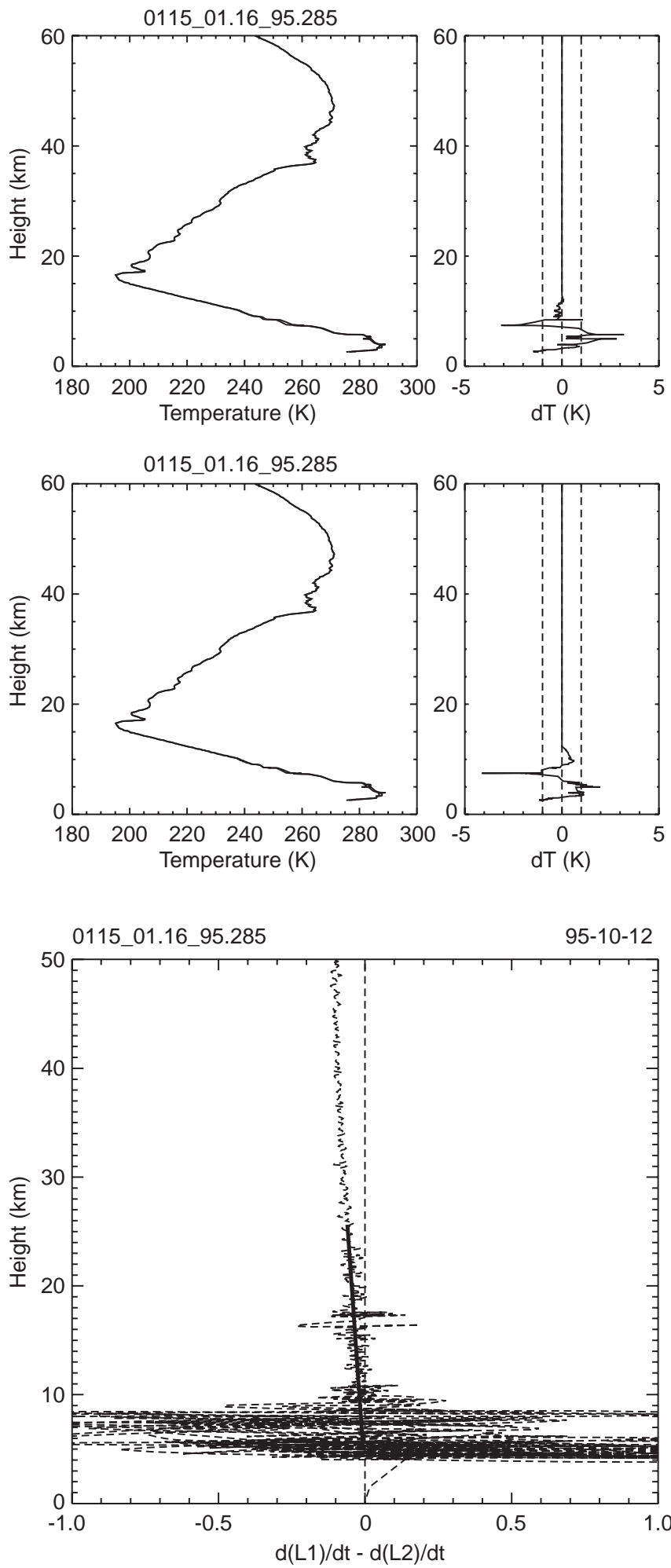

dary for subsequent refractivity retrieval. (The problem that biases may be introduced in this way is discussed in Sect. 4.)

Practically, we perform the combination by a heuristic statistical optimization approach using a variable weighting function depending on the GPS/MET data quality (Hocke, 1997). Heuristic means that though we based our optimization formally on the theory of
Fig. 3. Illustration of reliable ionospheric correction in the troposphere by means of extrapolating ionospheric Doppler shift linearly downwards to below $12.5 \mathrm{~km}$ height. The upper four panels show temperature retrieval results and temperature differences $(\mathrm{d} T)$, respectively (difference relative to direct $L 1-L 2$ combination for the entire profile). The reliable method (top panels) is illustrated and a straightforward method, which simply uses $L 1$ Doppler below $12.5 \mathrm{~km}$ height (middle panels). The bottom panel shows the ionospheric Doppler in terms of the L1-L2 Doppler difference (dotted profile), and indicates the Doppler extrapolation applied (solid line). The primary data are from GPS/MET occultation event 115 on October $12,1995,15: 12 \mathrm{UT}$, located at $1.1^{\circ} \mathrm{S}$ and $51.9^{\circ} \mathrm{W}$

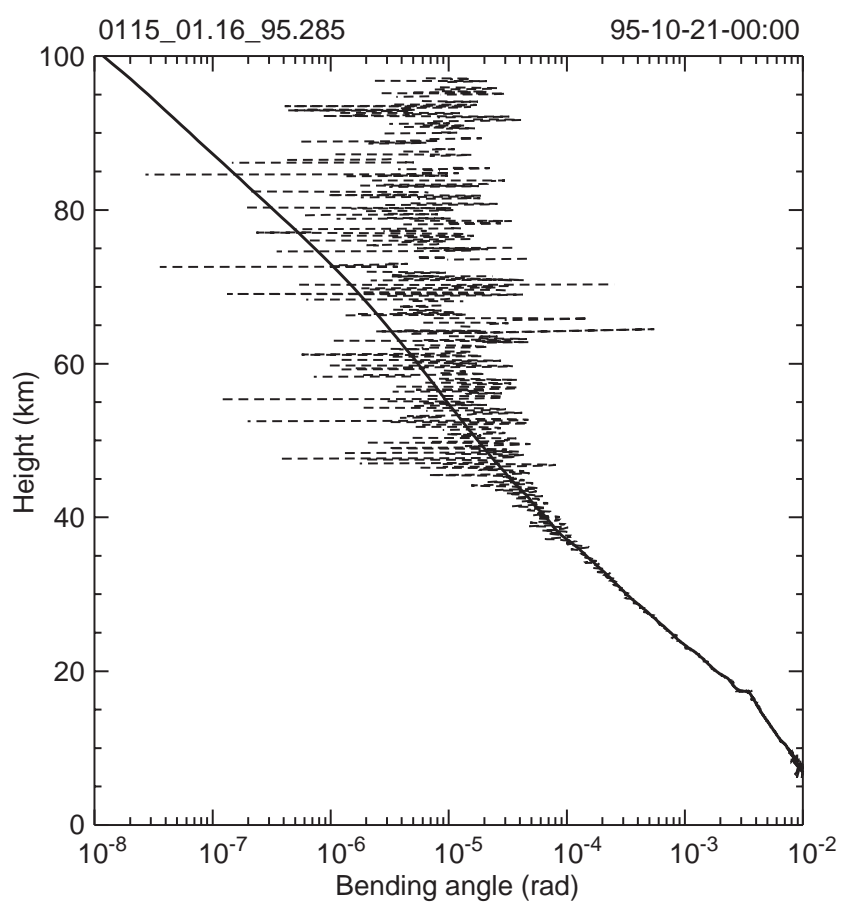

Fig. 4. A typical bending angle profile calculated from GPS/MET phase measurement data (dotted line) for the same occultation event as in Fig. 3. It is compared to a profile (solid line) obtained after a combination of the GPS/MET bending angle profile and the colocated MSISE-90 model bending angle profile using a statistical optimization approach

Gaussian random processes, our actual variance estimation does, for the sake of practical advantages, not strictly follow this theory. The combination is actually applied between $40 \mathrm{~km}$ height (currently a fixed value) and a variable height level of about $60 \mathrm{~km}$ (the level depending on the data quality of an individual event). Below the lower height level we use the measured bending angle data alone, and above the upper height level we use the model bending angle data alone.

3.2 Retrieval of the refractive index with a matrix inversion technique For an atmosphere with local spherical symmetry near the perigee of the occultation rays there exists an unique relationship between the atmospheric bending angle $\alpha(a)$ and the atmospheric refractive index $(n)$ as a function of radius $(r)$. Generally, 
the Abelian integral inversion method (e.g., Phinney and Anderson, 1968) is applied to convert bending angles into a refractive index profile (e.g., Hajj et al., 1995; Kursinski et al., 1996; Hocke, 1997).

For this study, we retrieved the refractive index profile not by integral inversion but by the application of a matrix inversion technique, which was in its basics introduced by Feng et al. (1996) at the 1996 GPS/MET workshop in Tucson, Arizona. The matrix inversion technique can be considered as a discrete representation of the inverse Abel transform. Though the technique as used here must lead to identical results as integral inversion, we applied it because it is more directly extendable to inversion, and characterization of the inversion, by optimal estimation (Rodgers, 1976, 1990), which we plan to implement in a future step.

The basic relation between bending angle $\alpha_{i}\left(a_{i}\right)$ of the $i$ th ray and the refractive index $(n)$ at the perigee location of the ray is given by the following integral equation, representing the forward Abel transform (e.g., Fjeldbo et al., 1971):

$\frac{\alpha_{i}}{2 a_{i}}=\int_{x=a_{i}}^{\infty} \frac{\mathrm{d}(\ln n)}{\mathrm{d} x} \frac{\mathrm{d} x}{\sqrt{\left(x^{2}-a_{i}^{2}\right)}}$.

Since the refractive index is close to unity in the terrestrial atmosphere, it is re-defined in terms of refractivity $(N)$ by

$\mathrm{N}=(\mathrm{n}-1) * 10^{6}$.

The unknown term $\mathrm{d}(\ln n) / \mathrm{d} x$ in Eq. 2 is replaced by the term $\mathrm{d} \tilde{n} / \mathrm{d} x$ with $\tilde{n}=N^{*} 10^{-6}$, taking into account Eq. 3. We then formulate, by dividing the atmosphere into $k$ layers with a layer thickness of $\Delta x_{k}$, and by assuming the gradient of the refraction index to be constant within each layer, the following discrete approximation of Eq. 2:

$\frac{\alpha_{i}}{2 a_{i}} \cong \sum_{k=1}^{i} \nabla \tilde{n}_{k} \frac{\Delta x_{k}}{\sqrt{\left(x_{k}^{2}-a_{i}^{2}\right)}}$.

Equation 4 can be re-written into the matrix formulation

$\overline{\left(\frac{\alpha}{2 a}\right)}=\overline{\bar{A}} \cdot \nabla \tilde{n}$,

where $\overline{\left(\frac{\alpha}{2 a}\right)}$ denotes the known data vector, $\overline{(\nabla \tilde{n})}$ the unknown parameter vector, and the matrix $\overline{\bar{A}}$ represents the (forward) Abel transform operator, containing the whole geometric information, which maps the parameter space into measurement space. The rows of the matrix correspond to the number of rays, $i$, and the columns correspond to the number of atmospheric layers, $k$. The operator matrix $\overline{\bar{A}}$ is composed of the matrix elements

$$
\begin{aligned}
A_{i k}= & \frac{\Delta x_{k}}{\sqrt{\left(x_{k}^{2}-a_{i}^{2}\right)}}=\ln \left|x_{k-1}+\sqrt[2]{x_{k-1}^{2}-x_{i}^{2}}\right| \\
& -\ln \left|x_{k}+\sqrt[2]{x_{k}^{2}-x_{i}^{2}}\right|
\end{aligned}
$$

where the right hand side expression is a pole-free form convenient for numerical use.

The generalized solution, for the unknown parameter vector, of the system of equations, Eq. 5, has the form

$\overline{(\nabla \tilde{n}})=(\overline{\bar{A}})^{-g} \overline{\left(\frac{\alpha}{2 a}\right)}$,

where $\overline{\bar{A}}^{-g}$ represents the generalized inverse (e.g., Press et al., 1992). For an overdetermined system (assuming a number of atmospheric layers smaller than the number of rays), the generalized inverse takes the form

$\overline{\bar{A}}^{-g}=\left(\overline{\bar{A}}^{T} \overline{\bar{A}}^{-1} \overline{\bar{A}}^{T}\right.$,

where $\overline{\bar{A}}^{T}$ denotes the transpose and $\overline{\bar{A}}^{-1}$ the inverse of $\overline{\bar{A}}$, and where the solution corresponds to the leastsquares solution of the overdetermined problem.

In our case, we have set up the matrix operator $\overline{\bar{A}}$ as a lower-triangular square matrix. The solution of this even-determined problem, for the refractive index gradients which are finally accumulated to yield the refractive index profile, can then directly utilize the inverse of the matrix $\overline{\bar{A}}$, i.e., $\overline{\bar{A}}^{-g}$ degenerates to $\overline{\bar{A}}^{-1}$. Alternatively, an efficient back-substitution algorithm is applicable to obtain the unique solution to this evendetermined problem (e.g., Press et al., 1992).

We start the inversion of the bending angle profile at a top height level of $110 \mathrm{~km}$ (above which vacuum can be reasonably assumed for our purposes) with the MSISE-90 model bending angle data. The layer thickness is decreasing with decreasing height which results in a tropospheric layer thickness of about one fourth of the stratospheric layer thickness. The effect of various height resolutions, or layer thicknesses, is demonstrated in Fig. 5, which shows a GPS/MET-derived temperature profile from October 21, 1995, at equatorial latitudes. In our data processing, we finally chose a most appropriate height sampling of about $200 \mathrm{~m}$ in the upper stratosphere decreasing to $50 \mathrm{~m}$ in the troposphere (solid line). This layer thickness contains most reasonably the whole information on atmospheric structures (as confirmed by independent Abelian integral inversion). In comparison, the same profile with a height sampling of about $100 \mathrm{~m}$ in the upper stratosphere (dotted line) already begins to approach numerical instability. On the other hand, with a height sampling of about $400 \mathrm{~m}$ in the upper stratosphere (dashed line), significant parts of the small-scale information are already lost.

\subsection{Calculation of atmospheric parameters}

In the third step of the data analysis, the atmospheric parameters refractivity $(N)$, density $(\rho)$, pressure $(p)$ and temperature $(T)$ are derived from the refractive index assuming dry air (since we do not analyze tropospheric data in this study). In the stratosphere, and in the cold parts of the troposphere with temperatures less than about $250 \mathrm{~K}$, water vapor is negligible. Starting values for refractivity and temperature, initializing the calculations at the top height level of $110 \mathrm{~km}$, are taken from 


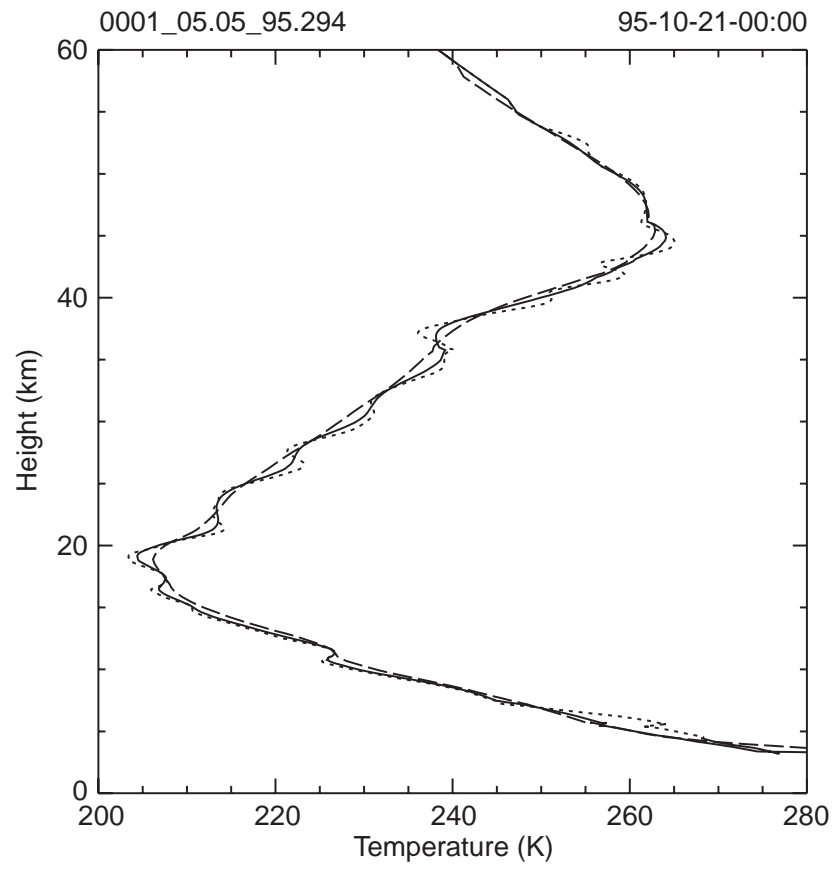

Fig. 5. The topic of height sampling in matrix inversion retrieval is illustrated by comparing three temperature profiles with different layer thicknesses adopted, all corresponding to the same occultation event 1 on October 21, 1995, 00:00 UT, located at $38.2^{\circ} \mathrm{N}$ and $139.2^{\circ} \mathrm{E}$. Profiles obtained with layer thicknesses (in the upper stratosphere) of about $400 \mathrm{~m}$ (dashed line), $200 \mathrm{~m}$ (solid line), and $100 \mathrm{~m}$ (dotted line) are shown

the MSISE-90 model. The following basically wellknown equations are used for the determination of the atmospheric parameters:

$$
\begin{aligned}
& N(h)=(n(h)-1) * 10^{6}, \quad \text { with } h=a / n(a)-r_{C} \\
& \rho(h)= C_{1} * N(h) \\
& p(h)=\int_{h}^{110 \mathrm{~km}} g\left(h^{\prime}\right) \rho\left(h^{\prime}\right) \mathrm{d} h^{\prime}, \quad \text { with } \\
& \quad g(h)=9.807 *\left(\left(r_{E} /\left(r_{E}+h\right)\right)^{2}\right. \\
& T(h)=C_{2} * \frac{p(h)}{N(h)}
\end{aligned}
$$

Here, $h$ is the geometric height, where $a / n(a)(=r)$ denotes the radius at the perigee of ray $(a)$ (Fig. 1), and $r_{C}$ denotes the Earth's radius of curvature in the occultation plane at the occultation location. $g(h)$ is the acceleration of gravity (latitudinal mean), where $r_{E}$ is the Earth's radius. The factors $C_{1}\left(=4.488 \cdot 10^{-3}\right)$ and $\mathrm{C}_{2}(=77.6)$ are constants determined by the refractive properties of air, its mean molar mass, and the universal gas constant (e.g., Kirchengast and Ladreiter, 1996; Hocke et al., 1997).

We confirmed the validity and quality of atmospheric profiles retrieved with the matrix inversion method with forward-inverse simulations based on atmospheric model data. We also compared, based on GPS/MET data, matrix-inverted profiles to profiles retrieved with the

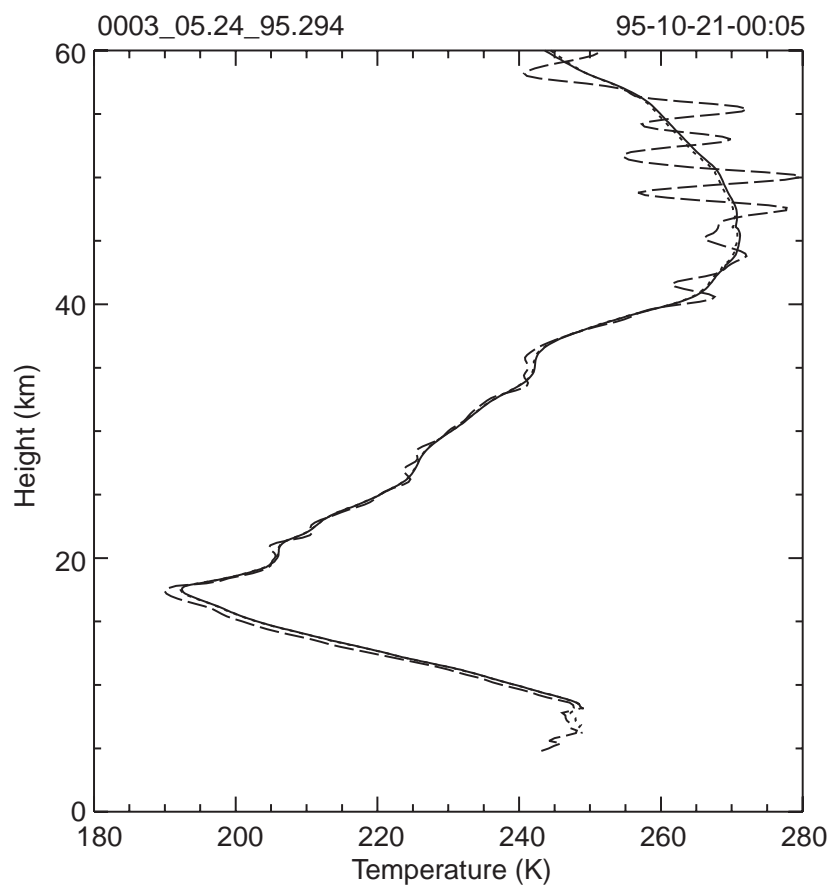

Fig. 6. Comparison of temperature profiles retrieved with Abelian matrix inversion (solid line), Abelian integral inversion calculated at the IMG/UoG (dotted line), and Abelian integral inversion calculated at UCAR, Boulder (dashed line). The GPS/MET data are from the occultation event 3 on October 21, 1995, 00:05 UT, located at $10.5^{\circ} \mathrm{N}$ and $136.7^{\circ} \mathrm{E}$

standard integral inversion. An example of the latter comparisons is shown in Fig. 6. The solid line denotes a GPS/MET temperature profile retrieved by the application of the matrix inversion technique. It shows practically no difference to the same profile retrieved using the Abelian integral inversion (dotted line, almost hidden by the solid line). The dashed line, for further comparison, denotes a profile calculated also with Abelian inversion at UCAR, Boulder, Colorado. This profile shows locally significant differences compared to the IMG/UoG profiles, especially in the upper stratosphere, which are mainly due to different upper-boundary initialization. The unrealistic temperature drop near the lower data boundary in the troposphere occurs since we did not take moist refractive contributions and diffractive effects into account.

\section{Error estimation analysis}

An error estimation analysis has been performed considering bending angle profile errors and the resulting errors in refractivity, pressure and temperature profiles. Total statistical errors as well as systematic errors due to upper-boundary initialization of the retrieval by a priori bending angles have been studied. Except for upper-boundary initialization, we do not explicitly consider any other potential source of systematic errors in this study of which primarily residual ionospheric errors under moderate to high solar activity conditions were of relevance. However, such residual 
ionospheric errors would lead to systematic errors qualitatively not different from those due to upperboundary initialization. Concerning details of the various error sources which may contribute to the total error in GPS occultation data, the recent work of Kursinski et al. (1997) provides a good overview discussion.

The most suitable type of data processing, and the corresponding error properties, depend on the retrieval purpose, i.e., the intended use of the retrieved profiles. For instance, for weather prediction purposes the individual profile is of special interest. It is then sensible to initialize the bending angle profile at high altitudes with a priori bending angle data, as described in Subsect. 3.1.3, in order to minimize the downward propagation of errors. This use of add-on bending angle data potentially introduces a systematic deviation in addition to the statistical errors, which has to be considered in the error estimation analysis. On the other hand, for a purpose such as climate monitoring, the individual profile is of less interest, since a large number of occultation events is averaged. Here it is not sensible to involve a priori data, potentially leading to systematic bias, except independent very good quality (high-altitude) data were available. Thus in this case just a statistical bending angle error has to be taken into account. However when analyzing occultation data without explicit a priori much care has to be taken to avoid systematic errors due to significant noise at high altitudes.

\subsection{Statistical error}

The analysis of the $50 \mathrm{~Hz}$-sampled GPS/MET bending angle data between $75 \mathrm{~km}$ and $95 \mathrm{~km}$ shows a mean magnitude of the residual bending angle noise of 10 to $20 \mu \mathrm{rad}$. This height range is chosen for estimating random noise, since it lies above significant neutral bending and at the same time below the influence of ionospheric (E-layer) bending. The random noise begins to exceed the bending due to the neutral atmosphere above about $50 \mathrm{~km}$ as can be seen in Fig. 4. This residual bending angle noise is partially caused by residual statistical ionospheric error. Other relevant contributions to the bending angle noise include thermal noise of the GPS receiver, local multipath, clock instabilities, and further noise incurred due to differencing techniques applied to the raw phase data (see Kursinski et al., 1997).

\subsection{Systematic error}

We assumed a "bad case" systematic error of $5 \%$ in the model bending angle, which corresponds to a systematic error of about $5 \%$ in the density in the MSISE- 90 model at altitudes above $50 \mathrm{~km}$. For the error estimation analysis, such a systematic 5\% error was added to the whole a priori model bending angle profile (baselined as true, if no error added), mimicking an overestimation of the actual atmospheric density (the biases obtained for corresponding underestimation are roughly mirror-symmetric). Basically, this "bad case" systematic model bending angle error produces a systematic bias in retrieved refractivity profiles of the order of 0.01 (in refractivity units) in the upper stratosphere, and of less than 0.01 in the lower stratosphere and troposphere. The systematic pressure bias lies between 0.1 and 0.2 mbar in the upper stratosphere, and increases to about $2.5 \mathrm{mbar}$ in the troposphere. The systematic temperature bias is near $5 \mathrm{~K}$ in the upper stratosphere, decreases to less than $2 \mathrm{~K}$ in the lower stratosphere, and is less than $1 \mathrm{~K}$ below $20 \mathrm{~km}$ height.

\subsection{Error estimation methodology}

We employed a Monte Carlo method for our error estimation analysis, since a covariance matrices-based error analysis, in principle a powerful tool for error characterization when using a matrix inversion technique (e.g., Rodgers, 1976, 1990), is very difficult to apply in this particular case involving extremely nonlinear high-altitude errors. For our specific case study, we took an arbitrarily chosen occultation event from October $12,1995,15: 12 \mathrm{UT}$ at $1.1^{\circ} \mathrm{S}$ and $51.9^{\circ} \mathrm{W}$. The statistical bending angle error for this reference profile was about $15 \mu \mathrm{rad}$, at $50 \mathrm{~Hz}$ sampling rate, which is very representative for GPS/MET profiles. The statistical rms errors in the retrieved refractivity, pressure and temperature profiles were determined by applying the Monte Carlo technique in form of 1000 trial retrieval runs with randomly generated Gaussian bending angle error (rms $15 \mu \mathrm{rad}$ ). The different rms errors were then calculated based on the ensembles of 1000 retrieved profiles. A small jitter, due to the finite ensemble size, on the final rms error profiles was smoothed with a 5-point sliding window (before producing the plot panels of Fig. 7 and Fig. 8).

\subsection{Results of error estimation analysis}

The results of our error estimation analysis are shown in Figs. $7 \mathrm{a}, 7 \mathrm{~b}$ and $8 \mathrm{a}, 8 \mathrm{~b}$, where each figure shows the bending angle error (upper left panel), refractivity error (upper right panel), pressure error (bottom left panel), and temperature error (bottom right panel). The different types of total error noted already were analyzed step by step in the error estimation and it is the essential results which are visualized by Figs. 7 and Fig. 8.

The "low end" step, illustrated in Fig. 7a, is the case of weakly filtered bending angle data (5 point-sliding window filter) with no application of statistical optimization. A corresponding initial statistical error of about $7 \mu \mathrm{rad}$ in the GPS/MET bending angle data leads to significant errors in the retrieved atmospheric profiles, such as a temperature error exceeding $3 \mathrm{~K}$ already below $30 \mathrm{~km}$ height, and $10 \mathrm{~K}$ in the upper stratosphere. As Fig. 7a further shows, the refractivity rms error incurred 

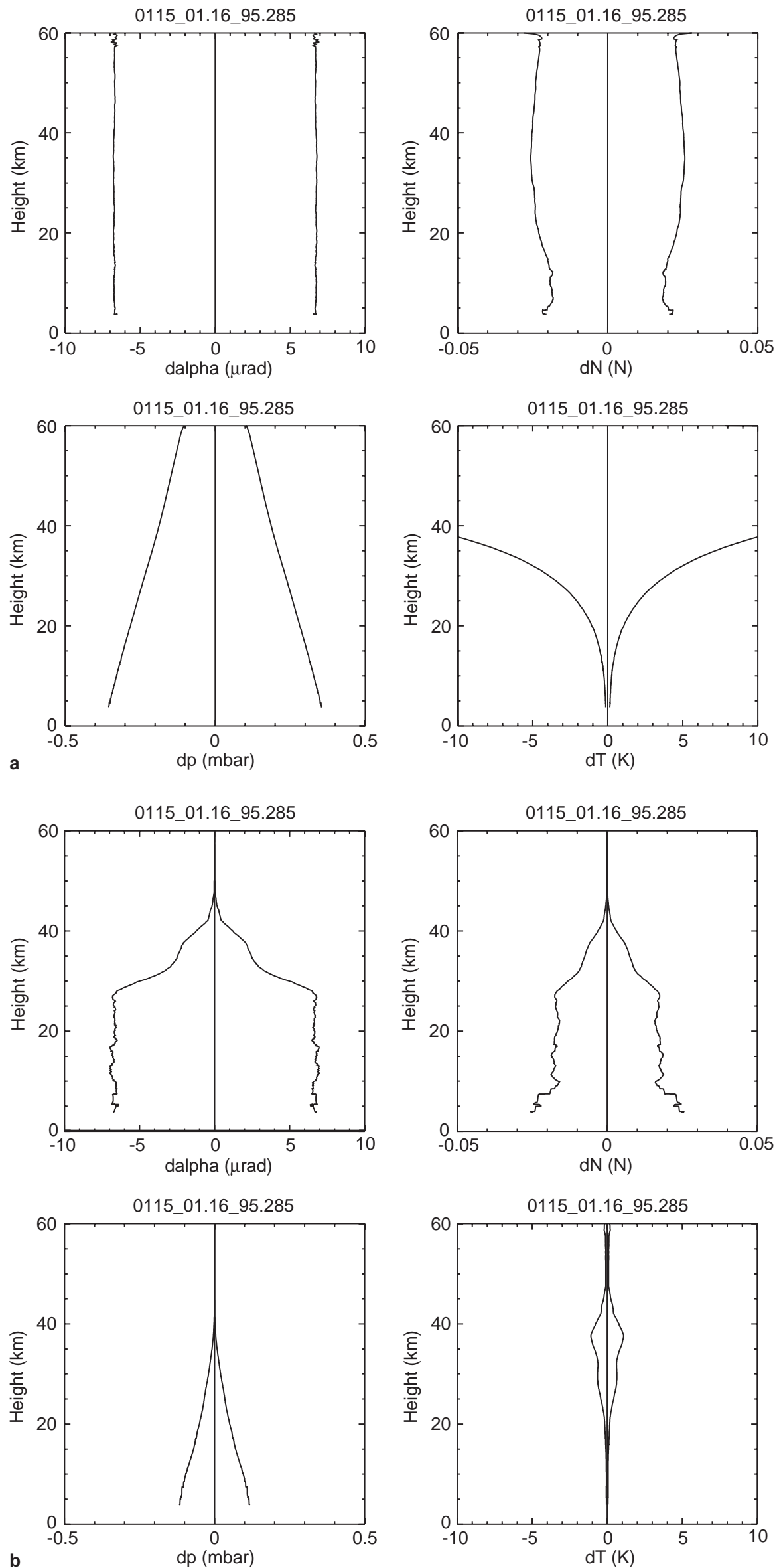
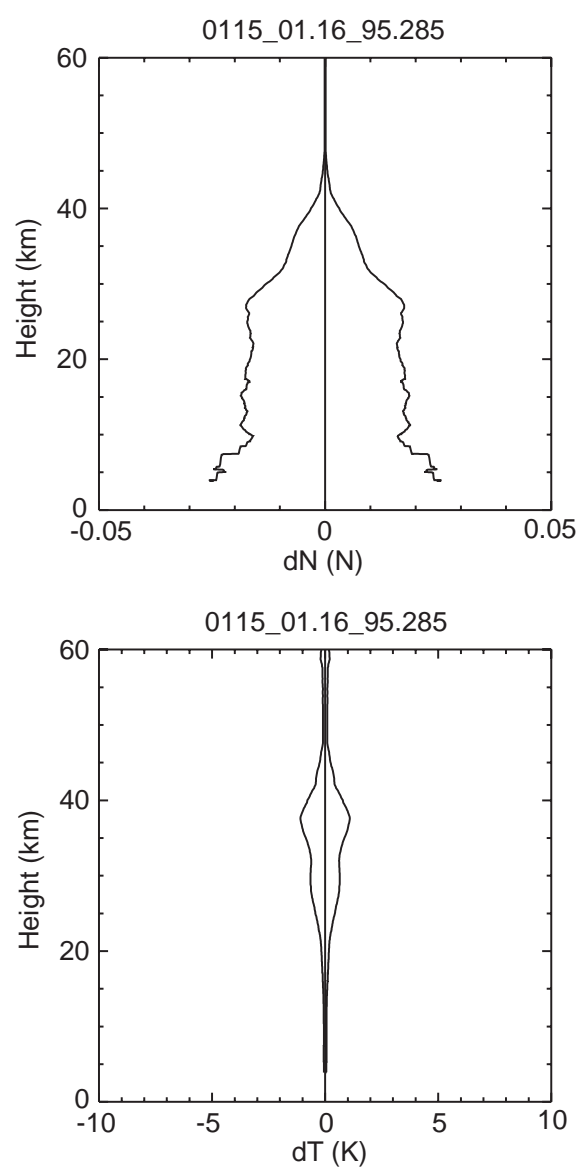

Fig. 7a, b. Results of error estimation analysis taking into account statistical bending angle error (upper left panel) only. The resulting statistical errors in refractivity (upper right panel), pressure (bottom left panel), and temperature (bottom right panel) are shown. a Shows the errors incurred if the $50 \mathrm{~Hz}$ bending angle data are weakly filtered only. More appropriate filtering of the bending angle data between $30 \mathrm{~km}$ and $70 \mathrm{~km}$ height plus upper-boundary initialization by perfect a priori data reduces the statistical error as shown in b. The GPS/ MET data used are the same as in Fig. 3 

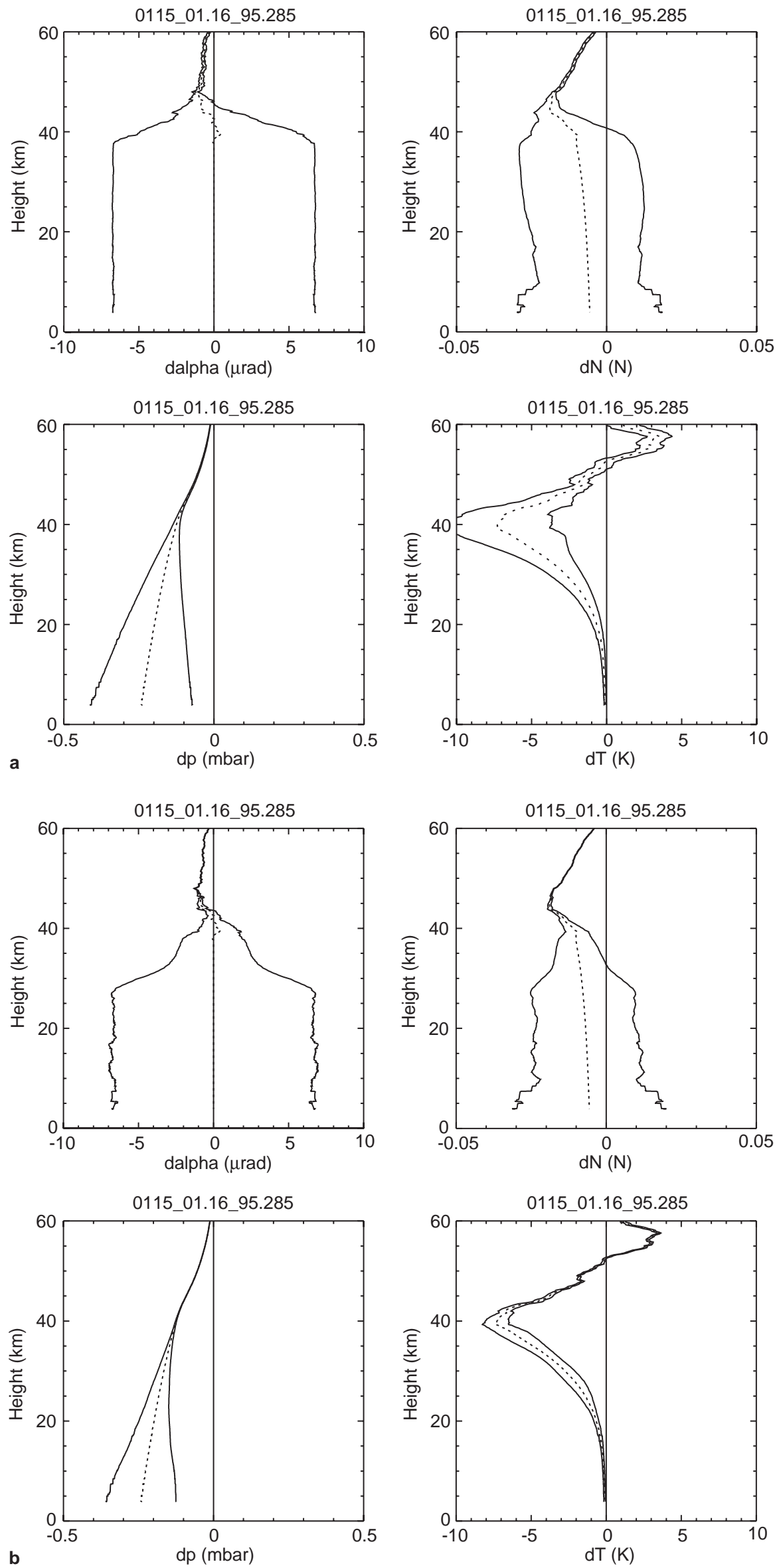

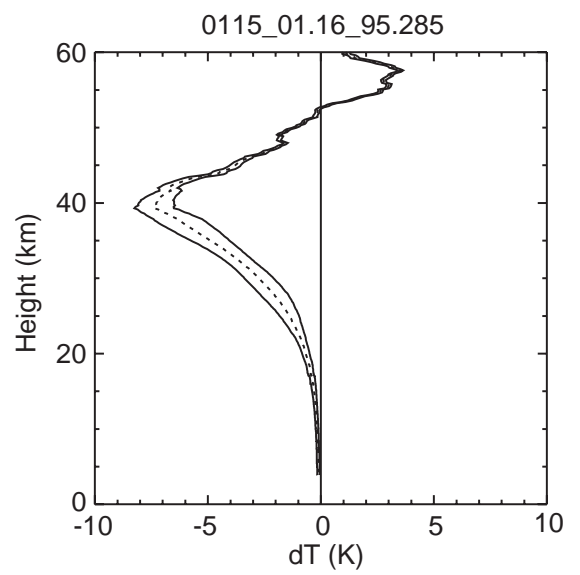

Fig. 8a, b. Results of error estimation analysis taking into account statistical bending angle error plus a systematic bending angle error of $5 \%$ in the a priori data used in the statistical optimization (upper left panel). The resulting errors in refractivity (upper right panel), pressure (bottom left panel) and temperature (bottom right panel) are shown. a Shows the errors incurred if the systematic error is applied and the GPS/MET bending angle data are weakly filtered only. Applying the same systematic error but more appropriate filtering of the GPS/MET bending angle data between $30 \mathrm{~km}$ and $70 \mathrm{~km}$ leads to the errors shown in b. The GPS/MET data used are the same as in Fig. 3 
is about 0.02 (in refractivity units), and the pressure error is about $0.4-0.2 \mathrm{mbar}$ in the lower stratosphere and $0.2-0.1$ mbar in the upper stratosphere, respectively. Note that for bending angle, refractivity, and pressure, which exhibit roughly exponential decrease with height, these weakly height-dependent absolute rms values correspond to significant relative errors at higher altitudes.

As illustrated in Fig. $7 \mathrm{~b}$, the situation drastically improves if the data processing includes appropriate filtering of the high-altitude errors (we added $\mathrm{Cos}^{2}$ filtering over 25 data points as noted in Sect. 3.1.3), and if upper-boundary initialization with high-quality a priori data helps control these errors (we applied our statistical optimization assuming perfect a priori data). In this most favorable scenario which highlights the potential of the occultation technique, the statistical bending angle error reduces to less than $2 \mu \mathrm{rad}$ in the upper stratosphere, which leads to a refractivity error of less than 0.02 there. The pressure error becomes less than about 0.1 mbar throughout and much less than this in the upper stratosphere. Finally, the temperature error becomes less than about $1 \mathrm{~K}$ in the upper stratosphere and significantly less than this in the lower stratosphere and troposphere. Applying just appropriate filtering without statistical optimization (not illustrated here), a step intermediate between those illustrated in Fig. 7a, b, essentially retains below $30 \mathrm{~km}$ height the favorable error characteristics shown in Fig. $7 \mathrm{~b}$ but increases significantly upper stratospheric errors. For instance, having then high-altitude bending angle rms errors of about $1.5-2 \mu \mathrm{rad}$ left, temperature errors begin to exceed $1 \mathrm{~K}$ at about $30 \mathrm{~km}$ and $3 \mathrm{~K}$ at about $40 \mathrm{~km}$.

In practice, as outlined earlier, the application of the statistical optimization will never lead to perfect initialization but the a priori bending angle data will introduce a bias, the strength of which depending on the accuracy of the a priori data. A "bad case" of such bias is illustrated by Fig. 8, which is rare if good high-altitude a priori data are available, but which will more or less frequently happen if just climatological model data such as from the MSISE-90 model are used.

Figure 8a shows the error estimation results when only weak filtering is applied to the GPS/MET bending angle data (as for Fig. 7a) and a systematic 5\% error is applied (added) to the a priori model bending angle data entering the statistical optimization. Significant bias is obviously incurred in the profiles (dotted lines), which has symmetrically superposed the statistical error (continuous lines). For instance, the pressure bias is about 0.2 mbar below $30 \mathrm{~km}$ height, and the temperature bias is generally $>2 \mathrm{~K}$ above $30 \mathrm{~km}$. The statistical error is quite reduced compared to Fig. 7a but still significant, since the filtering was weak.

Figure $8 \mathrm{~b}$ shows the estimation results when the systematic error applied is the same as for Fig. 8a but when appropriate high-altitude filtering is applied to the GPS/MET bending angle data (as for Fig. 7b). Here the bias (dotted lines) is of course unchanged but the statistical error is reduced to the level achieved for the favorable case of Fig. $7 b$.
We note that the exact bias behavior, in particular in the upper stratosphere, depends on the specific implementation of the statistical optimization (e.g., on our fixed lower optimization boundary of $40 \mathrm{~km}$ and the strong weight of the a priori data above about $50 \mathrm{~km}$ ). However, the general picture found for this behavior is qualitatively and quantitatively valid, as we found with various sensitivity checks. On the other hand, there is certainly moderate room left for reducing, by sensible improvement of our present implementation of statistical optimization, the "bad case" biases here obtained.

\section{Validation of GPS/MET data using ECMWF data}

GPS/MET-based refractivity and temperature profiles, retrieved by employing the processing chain described in Sect. 3, were validated through statistical comparison with atmospheric analyses obtained from the European Centre for Medium-range Weather Forecasts (ECMWF). For this purpose, we took all available GPS/MET level 2 data from October 20/21, 1995 (description in Sect. 2), and prepared ECMWF (dry air) refractivity and temperature data profiles corresponding to each occultation event in geographic location and time. The maps in Fig. 9 show the distribution and number of occultation events for October 20/21, 1995. The whole data set consists of 272 events, which was the total number of events available at level 2 for these two days of observation. The overplotted rectangular boxes (thick lines) circumscribe two regions of specific interest for our validation: a region over the Southern Pacific where it is known that observational data are sparse, and a region including Europe plus the USA, known to be well covered with observations. In particular, very few radiosonde data are acquired over the Southern Pacific, while many of them are available over Europe and the USA. Thus it is very reasonable to assume that the ECMWF data are closer to reality (largely radiosonde-driven) over Europe and the USA than over the Southern Pacific (largely model-driven due to lack of data) (see also Kursinski et al., 1996).

The ECMWF model data we used have a horizontal sampling of $1 * 1$ degree on a regular global latitude and longitude grid, and a vertical resolution of $31(\sigma$-)pressure levels corresponding to altitudes between $0 \mathrm{~km}$ and approximately $30 \mathrm{~km}$. Data from the highest level, corresponding to $10 \mathrm{mbar}$, were not used since this is the model boundary furnishing less realistic values. The resolution in time is $6 \mathrm{~h}$ and was linearly interpolated to $3 \mathrm{~h}$. The data set, from which all necessary parameters for GPS/MET validation can be derived, consists of temperature and humidity data for each vertical level, and of geopotential and pressure data corresponding to the surface level. The ECMWF profile selected as the one co-located in space-time with a given GPS/MET profile was always the "nearest neighbor" profile, i.e., the one of the $1 * 1$ degree-3 hours space-time grid which was nearest to the GPS/MET profile. This is a valid and 

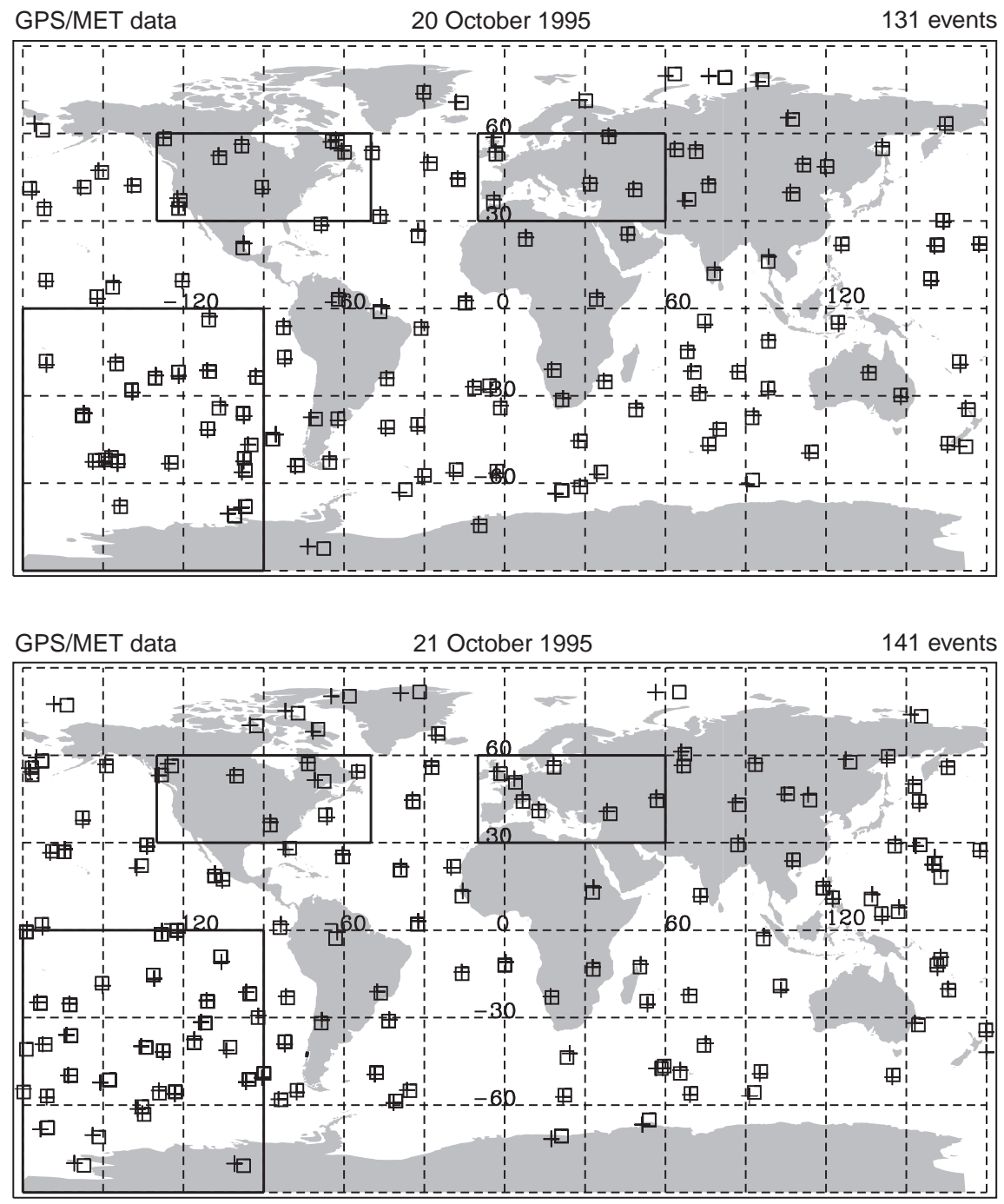

Fig. 9. Distribution and number of occultation events for October 20/21, 1995, used for the validation of GPS/MET data based on ECMWF data. The thick solid rectangles denote selected regions of good ECMWF data quality due to dense observational data (European plus USA area), and of degraded ECMWF data quality due to sparse observational data (South Pacific area). For each individual event, the perigee points of the topmost ray (center of the square symbol) and of the lowest ray (plus symbol) are indicated as taken from the "occultation tables" at the UCAR GPS/MET data server efficient selection for the type of statistical validation performed here.

\subsection{Statistical comparison of GPS/MET profiles with ECMWF profiles}

The parameters refractivity and temperature are compared in terms of altitude-dependent statistical differences. We interpolated the GPS/MET refractivity and temperature values to the ECMWF pressure level grid and then, using the ECMWF geopotential height information and an approximate Geoid, converted both the GPS/MET and ECMWF values to a standardized altitude grid consisting of 24 levels between $5 \mathrm{~km}$ and $25 \mathrm{~km}$. At the altitude grid, we computed difference profiles by subtracting the ECMWF data values from the GPS/MET data values. Mean differences $(\bar{x})$ the standard deviations of the differences $\left(\sigma_{x}\right)$, and the standard error of the mean differences $\left(\sigma_{\bar{x}}\right)$ for the whole data set are then computed using the following equations (with $M$ as the number of profile pairs):
$\bar{x}=\frac{\sum_{i=1}^{M} x_{i}}{M}$,

$\sigma_{x}=\sqrt{\frac{1}{(M-1)} \sum_{i=1}^{M}\left(x_{i}-\bar{x}\right)^{2}}$,

$\sigma_{\bar{x}}=\frac{\sigma_{x}}{\sqrt{M}}$.

Three different statistical comparisons are presented in Fig. 10a-c. The top panel always shows the number of occultation events, the middle panel shows refractivity comparisons $\left(N_{\mathrm{GPS}}-N_{\mathrm{ECMWF}}\right)$, and the bottom panel shows temperature comparisons ( $\left.T_{\mathrm{GPS}}-T_{\mathrm{ECMWF}}\right)$.

\subsection{Discussion of validation results}

Figure 10a illustrates the validation results of the full data set of 272 profiles distributed over the whole globe. The mean refractivity difference between 8 to $22 \mathrm{~km}$ altitude is less than $0.5 \%$, and increases to near $1 \%$ 

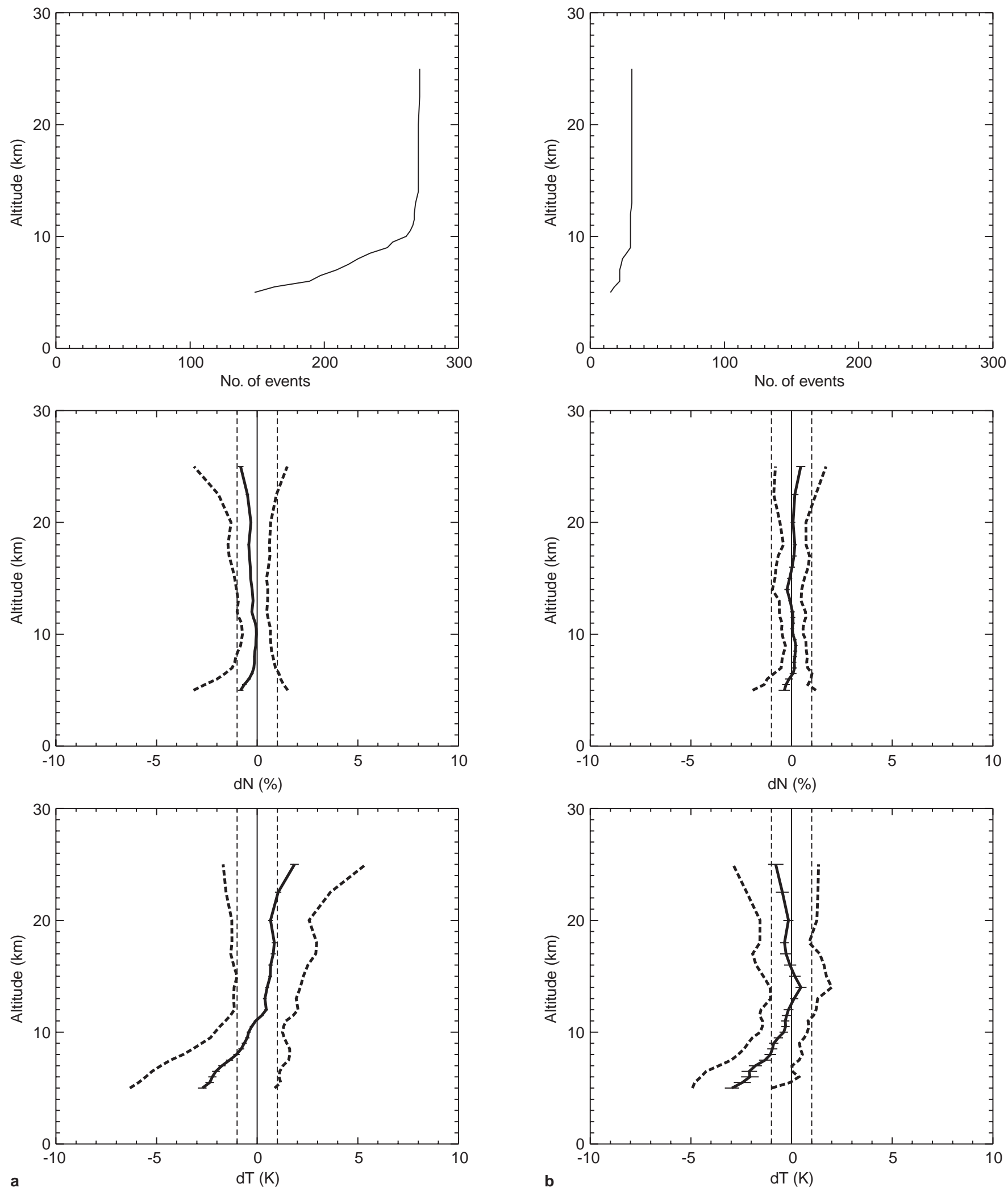

Fig. 10a, b. Results of statistical validation of GPS/MET data with ECMWF data. The top panels show the number of occultation events used (decreasing below $10 \mathrm{~km}$, since the lower boundary of individual events excludes tropospheric data of notably degraded quality). Refractivity differences $(\mathrm{d} N)$ are depicted in the middle panels, temperature differences $(\mathrm{d} T)$ in the bottom panels. The solid lines

denote the differences of means (bias profiles), the dotted lines denote the standard deviations of difference profiles, and the horizontal bars depict the standard error in the bias profiles. Validation results are shown for a the whole global data set of October 20/21, $1995 \mathbf{b}$, for the Europe + USA area c, and for the South Pacific area For continuation of Fig. 10 please see page 136 

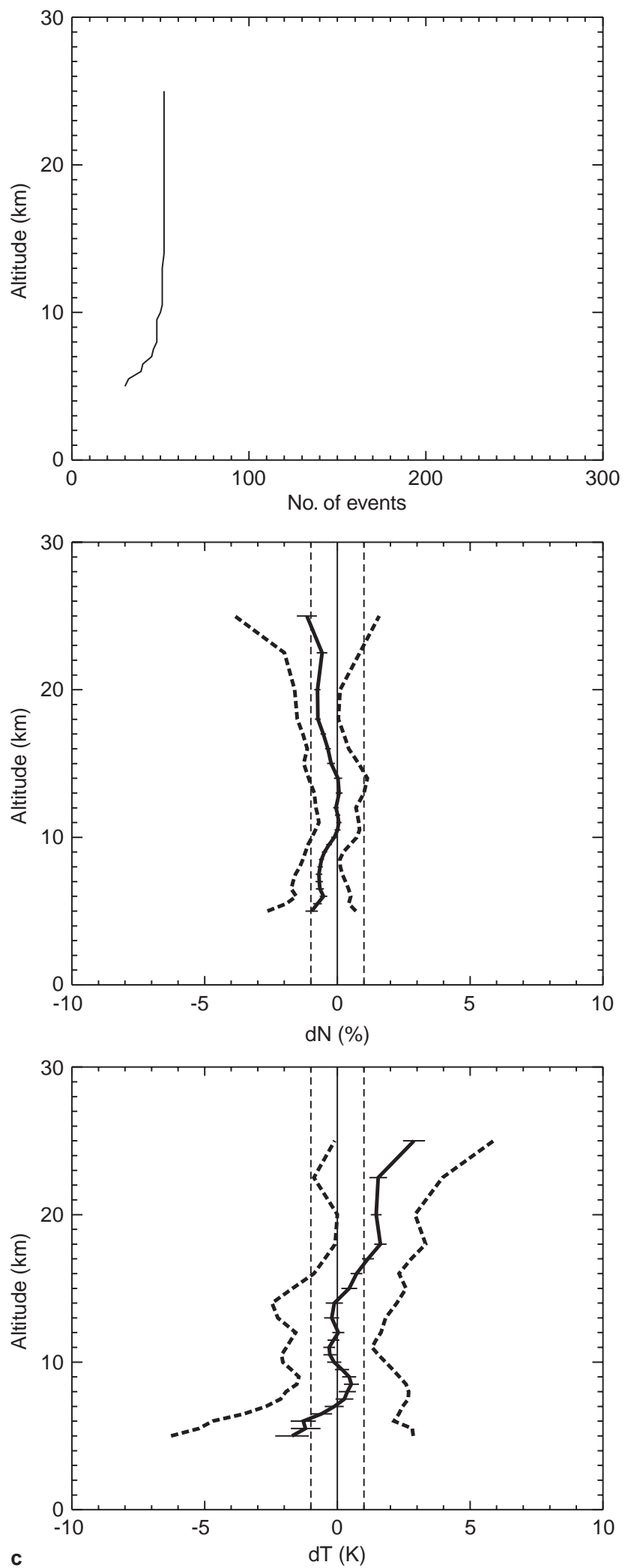

Fig. 10c (continuation of Fig. 10)

above and below. The mean temperature difference becomes less than $1 \mathrm{~K}$ between $8 \mathrm{~km}$ and $22 \mathrm{~km}$ altitude, and increases up to about $2 \mathrm{~K}$ above and below. The GPS/MET profiles are warmer than the
ECMWF profiles in the stratosphere/tropopause region but colder at tropospheric altitudes. The larger differences and larger standard deviations in the upper altitude range above about $20 \mathrm{~km}$ may be mainly due to the upper boundary influence of the ECMWF model; partly it may be also due to errors in the a priori information. In the lower tropospheric regions below $8 \mathrm{~km}$ the larger deviations may occur mainly due to the fact that they are calculated for a dry and basically smooth atmosphere ignoring moist contributions to refractivity and ignoring potential GPS/MET signal complexities typical for the first scale height above surface.

ECMWF analyses are supposed to be, as noted earlier, most accurate over areas like Europe and the USA, whilst GPS/MET accuracy can be assumed to be independent of geographic area. Thus comparison for such regions should minimize the contributions of ECMWF data to the joint variance in a comparison, allowing us to see the accuracy of GPS/MET data more directly than in a global data set. To check this we take the GPS/MET data set of 32 profiles available for a statistical comparison including these regions only (the maps of Fig. 9). The results are illustrated in Fig. 10b. In fact, the data consistency has significantly improved compared to Fig. 10a and the comparison indicates well the high accuracy achievable based on GPS occultation data. The mean refractivity differences are less than $0.25 \%$ between 8 and $22 \mathrm{~km}$, and the standard deviations less than $0.8 \%$. Mean temperature differences are less than $0.5 \mathrm{~K}$ between 10 and $22 \mathrm{~km}$ and the standard deviation is less than $1.5 \mathrm{~K}$, which confirms independent statistical validation work recently performed by Kursinski et al. (1996) and Rocken et al. (1997). Interestingly, temperatures are not improved below $10 \mathrm{~km}$ compared to the global validation, which we think points to an underestimation of the tropopause "coldness" by the ECMWF model (see Kursinski et al., 1996; Ware et al., 1996).

The sparse distribution of observational data over the Southern Pacific should lead to less reliable ECMWF data, which should in turn lead to degraded statistics when performing a GPS/MET-ECMWF comparison for this region only. We performed one using the 50 GPS/MET profiles available in the Southern Pacific region, and the results are illustrated in Fig. 10c. In fact, the consistency is now significantly degraded. Increased mean differences/standard deviations occur in both refractivity and temperature, e.g., now already above about $15 \mathrm{~km}$ altitude. For instance, above $18 \mathrm{~km}$, ECMWF profiles are in the mean about $1.5 \mathrm{~K}$ colder than GPS/MET profiles. This bias, which is obviously an underestimation of lower stratospheric temperatures by the ECMWF model, even largely explains a similar but smaller bias occurring in the whole data set (Fig. 10a) but not in the Europe + USA data set (Fig. 10b). The reason is that the number of occultation events in data sparse regions largely exceeds those in data dense regions (see Fig. 9). This also indicates well the potential of GPS occultation data to significantly improve, if properly assimilated into a numerical 
weather prediction model, forecasts and analyses of the atmospheric state.

\section{Summary and conclusions}

This study was concerned with different critical aspects of GPS/MET occultation data analysis, with a quantitative estimation of errors to be expected in the retrieved atmospheric profiles, and with a statistical validation effort aiming at checking the results recently derived by other authors. The GPS/MET data processing chain developed was introduced placing special emphasis towards open questions in ionospheric correction methodology and to the inversion of bending angle profiles to refractivity profiles, where we introduced a matrix inversion technique.

Ionospheric correction. We mainly studied, based on GPS/MET data, the impact of reduced $L 2$ sampling rates (which may be necessitated for retaining millimetric phase accuracy under GPS A/S-on conditions) and the correction of tropospheric data (which often are of complex structure due to a highly variable tropospheric refractivity field). Methods have been found, and demonstrated in case studies, which allow to effectively solve these specific problems. In case of reduced $L 2$ sampling rate it appears possible to retain sub-Kelvin accuracy up to $30 \mathrm{~km}$ height even for a $50 \mathrm{~Hz} @ L 1 /$ $1 \mathrm{~Hz} @ L 2$ sampling ratio. For ionospheric correction below the tropopause level a method appears attractive, where the $L 2$ Doppler data are replaced by $L 1$ Doppler data having added an ionospheric Doppler estimate extrapolated linearly downwards from the lower stratosphere. Despite such favorable results, more rigorous future studies on these and related topics (e.g., correction including auxiliary ionospheric data) are needed for improving ionospheric correction, especially since reliable ionospheric correction of occultation data will be highly relevant during the period of high solar activity due in the next few years.

Inversion of bending angle to refractivity. We have evaluated the quality of atmospheric profiles retrieved by employing a matrix inversion technique with forward-inverse simulations and with comparison to profiles retrieved with the usual integral inversion. Matrix inversion has been shown to lead to identical results as integral inversion, so that it is a fully valid alternative implementation of the inversion. Its advantage is that it is directly extendable to inversion by optimal estimation, which we plan to implement in the future. This then allows to optimally exploit the information in GPS occultation data in combination with suitable complementary data stemming from other (spaceborne) instruments or from dynamical models (in the variant of data assimilation).

Estimation of total errors. The quality of the GPS/ MET-derived atmospheric profiles (bending angle, refractivity, pressure, temperature) has been studied with an error estimation analysis employing a Monte Carlo technique. Total statistical errors as well as systematic errors due to upper-boundary initialization of the retrieval (by a statistical optimization approach) have been discussed. Better than $1 \mathrm{~K}$ temperature retrieval accuracy up to the stratopause is found for the most favorable case of properly smoothed statistical errors at high altitudes combined with perfect a priori data for initialization. Smoothed statistical errors alone allow $1 \mathrm{~K}$ accuracy up to $30 \mathrm{~km}$, but less than $3 \mathrm{~K}$ accuracy above $40 \mathrm{~km}$. For an unfavorable realistic case of imperfect initialization, assuming a systematic error of $5 \%$ in the a priori data, biases greater than $2 \mathrm{~K}$ were found to extend down to below $30 \mathrm{~km}$. For applications like weather prediction, upper-boundary initialization with a priori data seems a good choice, though it is important to invoke the best available complementary (spaceborne) high-altitude data; standard climatological model data are marginally useful only and may more or less frequently produce unfavorable individual retrievals. Great care has to be exercised in upper-boundary initialization for applications like climate monitoring, where any significant bias and drift needs to be avoided; if any, only reliable a priori data of high quality should be invoked in this case.

Statistical validation. Results of a validation of GPS/MET-derived refractivity and temperature profiles through comparison with co-located profiles from ECMWF analyses have been presented. The validation, performed with a total of about 270 globally distributed profiles and with selected subsets of this sample, confirmed the results recently derived by other authors. Clear indication of the high accuracy of occultation data was found in that very good agreement of upper troposphere/lower stratosphere refractivity and temperature data was found for a region (Europe + USA area), where the ECMWF analyses are known to be good (refractivity: $<0.8 \%$ rms error, $<0.25 \%$ bias; temperature: $<1.5 \mathrm{~K} \mathrm{rms}$ error, $<0.5 \mathrm{~K}$ bias). On the other hand, significantly poorer agreement was found for a region (Southern Pacific area), where the ECMWF analyses are known to be degraded due to sparse observational data (e.g., a temperature bias of about $1.5 \mathrm{~K}$ was found in the lower stratosphere, which is convincingly attributable to the analyses). These results indicate well the potential of GPS occultation data to become an extremely valuable data source for supporting applications like weather and climate modeling and prediction and climate change monitoring.

Acknowledgements. We are grateful to GPS/MET-PI R. Ware, GPS/MET project manager M. Exner, and the GPS/MET team at UCAR, Boulder, USA, for operating the GPS/MET experiment and supplying the data. We are also grateful to the European Centre for Medium-range Weather Forecasts (ECMWF) for supplying the ECMWF analyses for October 20/21, 1995. Furthermore, we thank S. Syndergaard from DMI, Denmark, for providing simulated occultation observables and for fruitful discussions on forward-inverse simulations, and $U$. Foelsche (IMG/UoG) for cooperation in preparing Fig. 9. This work was partially supported by the European Space Agency (ESA) under contract 11818/96/NL/CN. Partial support was also received from the K. \& M. Kaufmann Foundation, Graz, Austria.

Topical Editor F. Vial thanks A. S. Gurvich and A. Hauchecorne for their help in evaluating this paper. 


\section{References}

Born M., and E. Wolf, Principles of Optics, Pergamon Press, Oxford, UK, 1980.

Fjeldbo, G., and V. R. Eshleman, The bistatic radar-occultation method for the study of planetary atmospheres, J. Geophys. Res., 70, 3217-3225, 1965.

Fjeldbo, G., A. J. Kliore, and V. R. Eshleman, The neutral atmosphere of Venus as studied with the Mariner $\mathrm{V}$ radio occultation experiments, The Astron. J., 76, 123-140, 1971.

Feng, D., B. Herman, M. Exner, W. Schreiner, S. Sokolovskiy, D. Hunt, C. Rocken, R. Ware, and R. Anthes, GPS/MET temperature and pressure retrievals using constrained inversions, URSI GPS/MET Workshop, Tucson, AZ, Feb. 22-23, 1996.

Fishbach, F. F., A satellite method for temperature and pressure below 24 km, Bull. Am. Meteorol. Soc., 9, 528-532, 1965.

Gorbunov, M. E., Accuracy of the refractometric method in a horizontally nonuniform atmosphere, Izv., Atmos. Ocean. Phys., 5, 381-384, 1988.

Gorbunov, M. E., A. S. Gurvich, and L. Bengtsson, Advanced algorithms of inversion of GPS/MET satellite data and their application to reconstruction of temperature and humidity, Rep. 211, 40 pp., Max-Planck-Institut für Meteorologie, Hamburg, Germany, 1996.

Gurvich, A. S., and T. G. Krasil'nikova, Navigation satellites for radio sensing of the Earth's atmosphere, Sov. J. Remote Sensing, 7(6), 1124-1131, 1990.

Hajj, G. A., E. R. Kursinski, W. I. Bertiger, S. S. Leroy, T. K. Meehan, L. J. Romans, and J. T. Schofield, Initial results of GPS-LEO occultation measurements of Earth's atmosphere obtained with the GPS-MET experiment, paper presented at the IUGG XXI General Assembly, Int. Union of Geod. and Geophys., Boulder, CO, Jul. 2-14, 1995.

Hedin, A. E., Extension of the MSIS thermosphere model into the middle and lower atmosphere, J. Geophys. Res., 96, 1159-1172, 1991.

Hocke, K., Inversion of GPS meteorology data, Ann. Geophysicae, 15, 443-450, 1997.

Hocke, K., G. Kirchengast, and A. K. Steiner, Ionospheric correction and inversion of GNSS occultation data: problems and solutions, IMG/UoG Tech. Rep. for ESA/ESTEC No.2/97, 35 pp., Inst. of Meteorol. and Geophys., Univ. of Graz, Austria, 1997.

Kirchengast, G., and H. P. Ladreiter, The potential of the radio occultation technique based on GPS/GLONASS signals for determining fundamental atmospheric parameters (in German), Kleinheubacher Ber., 39, 677-686, 1996.

Kursinski, E. R., G. A. Hajj, W. I. Bertiger, S. S. Leroy, T. K. Meehan, L. J. Romans, J. T. Schofield, D. J. McCleese, W. G. Melbourne, C. L. Thornton, T. P. Yunck, J. R. Eyre, R, and N. Nagatani, Initial results of radio occultation observations of Earth's atmosphere using the global positioning system. Science, 271, 1107-1110, 1996.
Kursinski, E. R., G. A. Hajj, K. R. Hardy, J. T. Schofield, and R. Linfield, Observing Earth's atmosphere with radio occultation measurements using the Global Positioning System, J. Geophys. Res., 102, 23, 429-23, 465, 1997.

Ladreiter, H. P., and G. Kirchengast, GPS/GLONASS-sensing of the neutral atmosphere: Model independent correction of ionospheric influences, Radio Sci., 31, 877-891, 1996.

Lindal, G. F., G. E. Wood, H. B. Hotz, D. N. Sweetnam, V. R. Eshleman, and G. L. Tyler, The atmosphere of Titan: an analysis of the Voyager 1 radio occultation measurements, Icarus, 53, 348-363, 1983.

Lindal, G. F., D. N. Sweetnam, and V. R. Eshleman, The atmosphere of Saturn: an analysis of the Voyager radio occultation measurements, Astron. J., 90, 1136-1146, 1985.

Lusignan, B., G. Modrell, A. Morrison, J. Pomalaza, and S. G. Ungar, Sensing the Earth's atmosphere with occultation satellites, Proc. of the IEEE, 4, 458-467, 1969.

Melbourne, W. G., E. S. Davis, C. B. Duncan, G. A. Hajj, K. R., Hardy, E. R. Kursinski, T. K. Meehan, L. E. Young, and T. P. Yunck, The application of spaceborne GPS to atmospheric limb sounding and global change monitoring, JPL Publication 9418, 147 pp., Jet Propulsion Lab, Pasadena, CA, 1994.

Phinney, R. A., and D. L. Anderson, On the radio occultation method for studying planetary atmospheres, J. Geophys. Res., 73, 1819-1827, 1968.

Press, W. H., S. A. Teukolsky, W. T. Vetterling, and B. P. Flannery, Numerical Recipes in FORTRAN: the art of scientific computing, Cambridge University Press, Cambridge, UK, 1992.

Rocken, C., R. Anthes, M. Exner, D. Hunt, S. Sokolovskiy, R. Ware, M. Gorbunov, W. Schreiner, D. Feng, B. Herman, Y. H. Kuo, and X. Zou, Analysis and validation of GPS/MET data in the neutral atmosphere, J. Geophys. Res., 102, 29,849-29, 866, 1997.

Rodgers, C. D., Retrieval of atmospheric temperature and composition from remote measurements of thermal radiation, Rev. Geophys. Space Phys., 14, 609-624, 1976.

Rodgers, C. D., Characterization and error analysis of profiles retrieved from remote sounding measurements, J. Geophys. Res., 95, 5587-5595, 1990.

Vorob'ev, V. V., and T. G. Krasil'nikova, Estimation of the accuracy of the atmospheric refractive index recovery from Doppler shift measurements at frequencies used in the NAVSTAR system, Phys. Atmos. Oceans, 29, 602-609, 1994.

Ware, R., M. Exner, D. Feng, M. Gorbunov, K. Hardy, B. Herman, Y. Kuo, T. Meehan, W. Melbourne, C. Rocken, W. Schreiner, S. Sokolovskiy, F. Solheim, X. Zou, R. Anthes, S. Businger, and K. Trenberth, GPS Sounding of the atmosphere from Low Earth Orbit: preliminary results,, Bull. Am. Meteorol. Soc., 77, 1996.

Yunck, T. P., G. F. Lindal, and C. H. Liu, The role of GPS in precise Earth observation, paper presented at the Position, Location and Navigation Symposium, Orlando, FL, Nov. 29Dec. 2, 1988. 